\title{
Estudo Sobre Interação de Idosos em Redes Sociais Digitais
}

\section{Study on Senior Interaction in Social Networks Digital}

\section{Resumo:}

O presente trabalho apresenta um estudo sobre as interações sociais de grupos de idosos em uma rede social digital. $\mathrm{Na}$ análise qualitativa são estabelecidas as possíveis categorias de análise, utilizando a técnica de análise de conteúdo. $\mathrm{Na}$ análise quantitativa, é realizado o monitoramento dos dados das ações dos idosos associadas às funcionalidades da rede social digital Facebook, através de ferramentas computacionais e aplicado as métricas de redes sociais. Esse estudo tem como propósito melhor compreender as relações humanas dos idosos e avaliar a possibilidade de empregar a dinâmica da rede social como uma ferramenta on-line de auxílio para aprimorar as relações sociais dos idosos através da utilização da computação nas suas vidas. Como principal resultado desse estudo, constatou-se uma correlação entre a análise qualitativa e quantitativa, sendo comprovado que o grupo de idosos interagiu no mundo virtual utilizando a rede social principalmente para comunicação e socialização entre seus familiares e amigos.

Palavras-chave: Rede social digital. Interação social. Idosos.

\begin{abstract}
:
This work presents the studied of social interactions of a groups of seniors in a digital social network. The qualitative analysis sets out the possible categories of analysis, using the content analysis technique. In quantitative analysis is done a monitoring data of the actions of the elderly associated features of digital social network Facebook is performed through computational tools and applied the metrics of social networks. This study facilitates the improvement of human relations of the elderly and to assess the possibility of using the dynamics of the social network as an on-line tool to help improve the social relationships of older people through the use of computing in their lives. The main result of this study, there was a correlation between the qualitative and quantitative analysis and proved that the elderly group interacted in the virtual world using social network primarily for communication and socialization among family and friends.
\end{abstract}

Keywords: Social networking. Social interaction. Elderly.

CHEPE, Lucélia Moreira; ADAMATTI, Diana Francisca. Estudo Sobre Interação de Idosos em Redes Sociais Digitais. Informática na Educação: teoria e prática, Porto Alegre, v. 18, n. 2, p. 177-198, jul./dez. 2015.

\section{Lucélia Moreira Chepe}

\author{
Diana Francisca Adamatt
}

\section{Introdução}

0 presente trabalho apresenta um estudo das interações de um grupo de idosos através de uma rede social digital (RSD). Neste estudo, aborda-se tanto aspectos qualitativos como quantitativos. Os dados de natureza qualitativa são tratados através da análise de conteúdo (BARDIN, 2011 ou WASSERMAN; FAUST, 1994) visando estabelecer as possíveis categorias de análise. Os dados de natureza quantitativa são analisados por métricas para Análise de Redes Sociais (RECUERO, 2009, PAÚL, 2005) e capturados através das ferramentas computacionais, de forma a obter as ações mais realizadas pelos idosos associadas a funcionalidades apresentadas na RSD.

O envelhecimento atua diretamente em três principais aspectos da vida dos idosos: no 
biológico, a pessoa torna-se mais vulnerável; no social, que é relativo a mudança de papéis sociais e finalmente no psicológico que é a tomada de decisões e opções adaptando-se a senescência (NERI, 2009). Além desses aspectos, muitos idosos têm a diminuição das atividades sociais por vários motivos: falecimento de entes queridos, amigos e familiares, independência dos filhos, aposentadoria e viuvez (DOLL, 2006).

Entretanto, a satisfação de vida dos idosos é um sentimento individual, sendo que os fatores que influenciam esse bem-estar são a percepção subjetiva da saúde pela própria pessoa idosa e as relações sociais equilibradas e satisfatórias (WASSERMAN, 2012). Embora, as redes de relações pessoais sejam reduzidas com a idade avançada, são mais positivas com ênfase na qualidade preenchendo suas necessidades afetivas (DOLL, 2006). Além disso, os idosos possuem ampla experiência de vida e através da interação digital, podem ter uma nova fonte de conhecimentos e comunicação, ou seja, uma oportunidade de expressarem suas ideias e opiniões participando socialmente e contribuindo para a construção da sociedade.

Pesquisa recente do Comscore ${ }^{1}$ constatou que $84,2 \%$ dos brasileiros acima de 55 anos estão conectados no Facebook. Também, de acordo com os dados analisados, cada idoso gastou em média, 586 minutos na rede social, o que equivale a 19 minutos por dia. Segundo Wasserman et al. (2012), em um estudo realizado com um grupo de idosos da Universidade Federal do Rio Grande do Sul (UFRGS), mostram que a rede social predominante é o Facebook, representando $75 \%$ da preferência, seguido pelo Orkut com $11 \%$. Quanto a frequência no uso das RSDs, $64 \%$ dos idosos utiliza mais de duas vezes na semana.

${ }^{1}$ Disponível em: <http://asasesp.org.br/terceira.pdf>
No Brasil, os idosos possuem direitos assegurados em lei (FRAGOSO; RECUERO; AMA$R A L, 2011)$ à apropriação das tecnologias digitais. Portanto, esse estudo tem como objetivo analisar as redes sociais por meio de suas interações sociais em um grupo de idosos, para descobrir seus gostos, preferências e dificuldades, e formas de interação, visando compreender sua diversidade no ambiente digital das redes sociais e facilitar o aprimoramento das relações humanas dos mesmos através da tecnologia.

\section{Referencial Teórico}

O referencial teórico apresenta inicialmente a rede social, suas características e forma de representação. Ao longo desta seção é abordada também a interação social, tanto em nível de relações sociais mediada pela linguagem segundo a teoria interacionista vygotskiana, como em um ambiente digital mediado pelo computador. Por último, é apresentado o grupo social dos idosos, os aspectos do envelhecimento, o contexto e trabalhos relacionados.

\subsection{Rede Social}

O termo rede social existe há muito tempo e refere-se ao estabelecimento de relações sociais entre as pessoas. A RSD está relacionada as interações sociais estabelecidas no meio digital, identificáveis a partir das análises das interações (fluxos comunicacionais) entre os atores. Por sua vez sites de redes sociais são as plataformas, ou sistemas, que fornecem suporte para as redes sociais na Internet. Mais precisamente, pode-se definir um site de rede social como uma ferramenta on-line que permite aos usuários à construção de um per- 
fil identitário semipúblico (requer cadastro ou participação) ou semiprivado (requer convite ou aceitação), a publicação de suas redes sociais e a navegação por dentro dessas redes por outras pessoas (PAÚL, 2005, VYGOTSKY, 1998).

As RSDs estão fundamentadas na teoria dos grafos e são representadas por vértices ou nós, e arestas. Sendo assim, elas podem ser definidas através de dois elementos principais: os atores (nós) e as suas conexões (arestas) (RECUERO, 2009). Os atores seriam as pessoas, grupos ou instituições, representando os nós das redes. Eles não são facilmente identificados devido à distância que caracteriza a comunicação mediada pelo computador. Os atores são melhores definidos como representações performáticas dos indivíduos no ambiente do ciberespaço. As conexões são representadas por laços sociais, os quais se estabelecem através das interações sociais entre os atores. O conteúdo das interações define o tipo de relação social existentes entre os interagentes. Portanto: "A interação é a matéria prima das relações e dos laços sociais [...]" (PAÚL, 2005). Sendo assim, é necessário analisar a importância da interação social para o desenvolvimento humano e os processos interativos envolvidos no mundo digital.

\subsection{Interação Social}

Quando se afirma que o homem é um ser social significa dizer que ele vive em constante interação com o meio. A interação é base da vida social. As pessoas são seres formados através de processos interativos sociais, os quais possibilitam a construção das suas relações sociais, afetivas, profissionais, que servem de base para estruturação da vida pessoal (CASTELLS, 2003).
Na teoria interacionista vygostikiana, o desenvolvimento humano não é um produto de fatores biológicos, sociais e culturais analisados de forma isolada. Pelo contrário, as interações sociais (que são estabelecidas pelo contato social e a comunicação) produzem como resultado uma modificação no comportamento das pessoas que interagem. Essa modificação, além de alterar o meio em que se vive, também produz alterações nos indivíduos envolvidos. A interação social é essencial para o processo de aprendizagem e desenvolvimento humano.

Além disso, segundo Vygotsky (1998), o aprendizado é contínuo em todas as fases da vida. Para explicar o processo de aprendizagem, ele desenvolveu o conceito de Zona de Desenvolvimento Proximal (ZDP) e definiu que o ser humano possui dois níveis de desenvolvimento:

Nível de desenvolvimento Real (NDR): é a capacidade da pessoa de resolver um problema de forma independente, representa aquele conhecimento que já é dominado, são as funções que já se desenvolveram.

- Nível de desenvolvimento Potencial (NDP): é a capacidade da pessoa de resolver um problema com ajuda de uma pessoa mais experiente ou capaz, representa aquele conhecimento que não é dominado, são as funções que possuem as bases necessárias para serem desenvolvidas.

A Zona de Desenvolvimento Proximal refere-se à distância entre o NDR e o NDP. Sua criação é dinâmica e ocorre em um meio social com a mediação dos símbolos, sendo o principal a linguagem. Para Vygotsky (1998), não deve esperar as estruturas mentais da criança se formar para a aprendizagem, afirmando que o ensino é o responsável por desencadear a formação das estruturas mentais necessárias 
para a aprendizagem. É necessário, no entanto, não ultrapassar a capacidade cognitiva de cada ser humano, sempre respeitando a ZDP. Através desse importante conceito, é possível afirmar que o incentivo ao diálogo, a interação social e cultural e a criação de novas condições para aprendizagem por pessoas mais capazes são formas de intervenção mais eficiente na ZDP as quais possibilitam ampliar o aprendizado potencial.

Embora Vygotsky (apud CASTELLS, 2003) somente refira-se ao desenvolvimento do ser humano citando a criança como exemplo, afirma também que o aprendizado é contínuo em todas as fases da vida. Dessa forma, a pessoa idosa continua o desenvolvimento das suas capacidades intelectuais através das interações sociais. O contato social com outras pessoas, mediado por alguém com mais capacidade, permite, mesmo em uma idade avançada, que a pessoa, partindo do desenvolvimento real, consiga alcançar o desenvolvimento potencial. Esse processo de aprendizagem, além de promover as funções cognitivas, contribui também para um envelhecimento bem-sucedido.

Portanto, para construção efetiva do ser humano, além dos fatores biológicos, os aspectos sociais e culturais são fundamentais porque influenciam o desenvolvimento intelectual das pessoas. É através da interação social mediada pela linguagem que o indivíduo aprende a socialização no ambiente, adquirindo hábitos e costumes que se agregam a sua personalidade conseguindo dessa forma desenvolver sua capacidade intelectual, integrar-se na sociedade e construir sua própria identidade. A aquisição do conhecimento e a aprendizagem ocorrem na construção contínua do ser humano em sua relação com o meio em que vive (CASTELLS, 2003).

\subsection{Processos Interativos Mediados Pelo Computador}

A Internet engloba uma grande revolução tecnológica e tornou-se o principal marco da atual Era da Informação, com a mesma importância que a eletricidade foi para a Era industrial. A forma de comunicação da sociedade contemporânea foi alterada, permitindo a comunicação de muitos para muitos em uma escala global (PRIMO, 2000). Por sua vez, o acesso as informações na Internet foi facilitado e difundido para um número cada vez maior de pessoas através dos recursos da WorldWideWeb (Web).

A $W e b$ evoluiu muito rapidamente passando de passiva (Web 1.0) para ativa (Web 2.0) e tornando-se um dos principais espaços de trocas virtuais de informações no mundo. Portanto, como objeto deste estudo são as interações sociais em uma rede digital que ocorrem por intermédio das funcionalidades da Web 2.0, é importante analisar os processos de interação mediados pelo computador relacionando-os a evolução dessa ferramenta.

Segundo Primo (2000), a interação através do computador não pode ser somente reduzida a transmissão de informações, mas focada no que se passa entre os interagentes. Assim, de acordo com o relacionamento mantido entre os agentes envolvidos em uma interação mediada por computador, podem-se observar dois grandes grupos distintos de processos interativos:

- Interação Reativa: caracterizada por um universo fechado de relações determinadas por pares estímulo-resposta e ação-reação. Este tipo de interação apresenta relações lineares e pré-estabelecidas, ou seja, existe um conjunto de ações possíveis aguardando para serem realizadas. 
- Interação Mútua: com maior caráter dialógico, caracterizada por um universo aberto com relações interdependentes. O relacionamento está em constante desenvolvimento e evolui a partir de processos de negociação entre os interagentes. Os sujeitos participam ativamente da construção do relacionamento e se afetam mutuamente, recriando o processo a cada troca.

De acordo com os tipos de processos interativos envolvidos na comunicação mediada por computador definidas por (BLATTMANN; SILVA, 2007), pode-se estabelecer uma analogia com a $W e b$. Para isso é necessário analisar as definições e características da Web 1.0 e Web 2.0.

A Web 1.0 foi a primeira geração da Web. Blattmann e Silva (apud SCHNEIDER; IRIGARAY, 2008) relatam que nesta fase da Web, o conteúdo on-line dos sites era estático sem permitir a interação dos internautas. Predominava a publicação de informações e o uso de uma linguagem hipertexto como documentos de texto estáticos, com links entre si. Os internautas, durante a leitura, estabeleciam a trajetória não linear que mais Ihe interessava através do uso de links ou hiperlinks, porém precisavam escolher entre as alternativas pré-estabelecidas caracterizando processos de interação reativa mediada por computador.

Atualmente, a Web apresenta-se muito diferente, suportando o uso de sons, imagens, vídeos e mais uma diversidade de conteúdos multimídia. Além disso, as páginas $W e b$, antes estáticas, hoje se apresentam como dinâmicas, caracterizando a Web 2.0. Blattmann e Silva (apud SCHNEIDER; IRIGARAY, 2008). Nesta segunda fase da Web (Web 2.0), o conteúdo é alterado sem a interferência direta de um desenvolvedor, ou seja, ocorreu uma mudança no foco passando da publicação para participação. O diálogo e a construção do conhecimento de maneira coletiva passaram a ser mais valorizados, confirmando a tendência relatada por Primo (apud BLATTMANN; SILVA, 2007) que a Web 2.0 oferecerá cada vez mais suporte para interação mútua mediada por computador.

$\mathrm{Na}$ Web 2.0, representada principalmente pela interação mútua, os usuários deixam de serem receptores passivos para tornarem-se agentes de disseminação de informações através de Chats, Microblogs, Blog, Sites de Redes Sociais, etc. Nesta nova Web, mais colaborativa e interativa, devido a presença de usuários ativos no processo de comunicação, a rede social objeto desse estudo constitui-se como suporte desta nova concepção.

\subsection{Idosos}

O processo de envelhecimento somente pode ser compreendido analisando a relação em três diferentes aspectos fundamentais: biológico, psicológico e social. O envelhecimento é uma experiência heterogênea e, ao mesmo tempo, de vivência individual que não pode ser medido somente através da idade cronológica, mas levando em conta a integração entre as experiências passadas, presentes, expectativas futuras e o contexto social e cultural do indivíduo (MORAES; MORAES; LIMA, 2010).

$O$ envelhecimento biológico, também denominado de senescência, é um processo ativo e irreversível, representado por sinais de deficiência funcional que aparecem de forma gradual ao longo da vida (IBGE, 2013). Entre as mudanças apresentadas no decorrer desse processo, pode-se citar: a audição diminui ao longo dos anos; a visão declina para objetos próximos; o peso e o volume do encéfalo diminuem pela perda dos neurônios, mas as fun- 
ções mentais são preservadas até o final da vida (MORAES; MORAES; LIMA, 2010).

Por sua vez, o envelhecimento psicológico refere-se as capacidades psicológicas (percepção, aprendizagem e memória). Durante o processo de envelhecimento, as capacidades cognitivas, como a rapidez de aprendizagem e memória, diminuem naturalmente com a idade. Porém, essas perdas podem ser compensadas com ganho em conhecimento, sabedoria e experiência. Além disso, o idoso não perde a capacidade de raciocínio e não apresenta declínio de suas funções intelectuais com o passar do tempo (MORAES; MORAES; LIMA, 2010).

Por fim, o envelhecimento social é caracterizado por um processo de mudança de papéis sociais e refere-se ao grau de adaptação do individuo no desempenho desses papéis, bem como o comportamento esperado por pessoas da sua idade em uma determinada cultura e sociedade no qual esteja inserido. Socialmente, caracteriza o momento que a pessoa afasta-se do mercado de trabalho. Com a aposentadoria, ocorre uma redução de forma abrupta das relações sociais, redução salarial e falta de atividades fora do ambiente de trabalho (MORAES; MORAES; LIMA, 2010).

Sendo assim, o processo de envelhecimento é individual e heterogêneo. Além disso, para sua compreensão é necessário entender a relação existente entre os diferentes aspectos biológicos, psicológicos e sociais do envelhecimento e também os fatores sociais e culturais de cada indivíduo. Como afirma Vygotsky (apud CASTELLS, 2003), a construção contínua da identidade do ser humano é fruto de todos esses fatores analisados de forma conjunta. Portanto, influenciam a forma como as pessoas com idade mais avançada interagem com a sociedade.

Além disso, na sociedade contemporânea, o grupo representado pelos idosos aumentou sua população de forma significativa nas últimas décadas. No Brasil, através do censo 2010 realizado pelo Instituto Brasileiro de Geografia e Estatística (BRASIL, 2003) foi constatada a tendência de envelhecimento da população brasileira $O$ censo apresenta uma diminuição na proporção de jovens e um aumento na de idosos. A população com 65 anos ou mais, que era de $4,8 \%$ em 1991, passando a 5,9\% em 2000 e chegando a 7,4\% em 2010. As regiões Sul e Sudeste são as que apresentam as maiores proporções de idosos na população total, conservando a posição das regiões mais enveIhecidas do país. Aliado a isso, desde a década de 70 o Brasil apresenta acentuada queda de fecundidade. $O$ grupo de crianças de zero a quatro anos que representava 5,5\% em 1991, diminui para 4,7 em 2000 e chegando a 3,6 em 2010.

Outro resultado mais recente faz parte da pesquisa Tábuas de Mortalidade 2010 - Brasil, Grandes Regiões e Unidades da Federação (Revisão 2013), realizada também pelo Instituto Brasileiro de Geografia e Estatística (PÁSCOA, 2012), o qual revela que a expectativa de vida do brasileiro aumentou 11,24 anos de 1980 (62,52 anos) a 2010 (73,76 anos). Nesta pesquisa do IBGE (2013), na análise por região, verificou-se que a região Sul registra as mais altas taxas de expectativa de vida do país. Em 1980, correspondia a 66,01 anos, apresentando a taxa mais alta de expectativa de vida naquele ano. No ano de 2010 a região Sul alcançou (75,84 anos), seguida da Sudeste $(75,40)$, Centro-Oeste $(73,64$ anos $)$, Nordeste $(71,20$ anos) e Norte (70,76 anos). Devido principalmente aos avanços da medicina, as pessoas estão chegando a uma idade mais avançada, fato que evidencia a necessidade de uma maior preocupação com esta faixa etária. 


\subsection{Trabalhos Relacionados}

Atualmente as RSDs representam as plataformas digitais mais abrangentes para comunicação e socialização das informações no mundo virtual. Segundo Páscoa (apud LEWIS; ARIYACHANDRA, 2011), que apresentou um estudo de caso na Universidade Sénior Albicastrense, analisando a contribuição do Facebook na promoção do envelhecimento ativo. Foi possível constatar que os idosos consideram o Facebook uma ferramenta digital facilitadora e muito útil nas suas relações, visando um envelhecimento ativo porque "[...] aumenta a qualidade de vida, combate o isolamento, promove a socialização, é um complemento de lazer e entretenimento e aproxima gerações em que os avós e netos falam a mesma linguagem [...]" (PÁSCOA, 2012, p. 41). Na pesquisa, os idosos encararam o Facebook como um projeto de vida para envelhecer melhor porque se sentiram infoincluídos no meio digital, com maior participação na sociedade e criando valor para a comunidade.

Outros estudos foram desenvolvidos no sentido de analisar o uso das RSDs pelos idosos. Segundo Wasserman et al. (2012), a possibilidade de comunicação mais rápida com amigos e parentes em qualquer lugar do mundo, sem a necessidade de deslocamento, incentiva a utilização dessa ferramenta por essa faixa etária. Além disso, o estudo menciona as RSDs como um meio dos idosos serem inseridos no meio digital. Lewis (apud JANTSCH et al., 2012) pesquisou sobre o uso das RSDs e também constatou que elas possuem potencial de enriquecer a vida dos idosos, aumentando a qualidade de vida porque funciona como um meio facilitador da interação dos mesmos com a comunidade.
Pode-se citar também o documento integrante da pesquisa sobre a utilização das redes sociais pelos idosos, o programa Sociedade $\mathrm{Ci}$ vil da RTP2 em Portugal, que abordou o tema Redes Sociais Para Maiores de 50, de abril de 2013. Vários profissionais foram convidados para entrevista e no transcorrer do programa foi mencionado que os idosos estão cada vez mais presentes na Internet, mas a maioria está iniciando seu uso nas redes sociais. Como os idosos geralmente possuem mais tempo disponível, as redes sociais contribuem para aproximar mais as famílias (avós, pais e netos) que estão geograficamente distante e dessa forma os familiares encontram-se mais perto virtualmente. Quanto à questão da melhoria da qualidade de vida, para os idosos que possuem mobilidade reduzida as redes constituem um importante mecanismo de participação política e cívica, possibilitando estarem presente virtualmente nos acontecimentos sociais.

Segundo Jantsch et al. (2012), que analisaram as redes sociais e a sua influência na qualidade de vida dos idosos, as RSDs são utilizadas pelos idosos principalmente para comunicação seguido pelo lazer e entretenimento. As principais vantagens do uso das redes seriam a troca de informações e a possibilidade de reencontrar pessoas do passado. Além disso, o estudo concluiu que as redes sociais digitais possibilitam a construção de relacionamentos concretos com pessoas desconhecidas a partir de relações sociais estabelecidas no meio virtual constituindo uma forma de combater a solidão social e influenciando de forma positiva a qualidade de vida porque aproxima os idosos da sociedade e do mundo através da informática.

2 Disponível em: <http://www.rtp.pt/play/p1043/e114569/ sociedade-civil-viii $>$ 
Em outra pesquisa importante, Brandão e Silveira (2010) analisaram a forma como os jovens representam os idosos nas comunidades do Orkut que abordam essa temática, por considerarem a rede social um local onde as manifestações dos sujeitos parecem ser menos sujeitas a censura. Neste estudo, foram analisadas descrições, comentários e narrativas produzidas tanto em comunidades que se referem aos idosos de forma preconceituosa e pejorativa caracterizadas pela expressão eu odeio, como também aquelas que o representam de maneira benevolente, com demonstração de carinho, admiração e respeito. Foi constatado que a cultura do ser jovem apregoada pela sociedade contemporânea, aliada ao distanciamento dos avós ou de outras pessoas idosas, contribui muito para as representações negativas. Por outro lado, o contato maior com os idosos principalmente nas relações familiares além de apresentar mais membros nas comunidades foi muito significativo para as representações positivas do processo de enveIhecimento.

\section{Metodologia}

Nesta seção é apresentada a metodologia que foi adotada no presente trabalho. Primeiramente, é realizada a pesquisa sobre o estado da arte do tema. Em seguida, é definida a rede social digital selecionada para estudo. Logo após, são definidos os passos (Figura 1) visando a produção dos dados da pesquisa, bem como a análise dos mesmos:
Figura 1 - Metodologia da Pesquisa

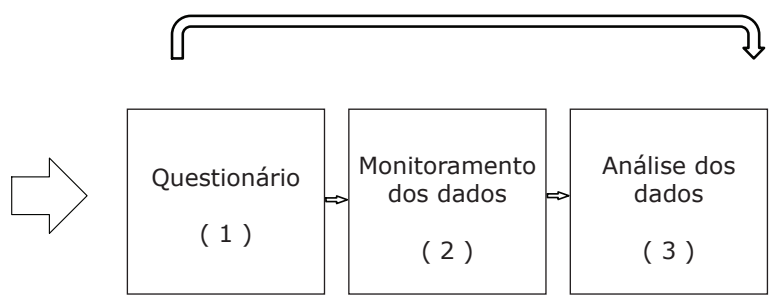

Fonte: Elaborada pelo pelas próprias autoras.

O primeiro passo consiste em um questionário (Quadro 1) no qual são abordados tanto questões de múltipla escolha como discursivas. A aplicação desse instrumento de coleta de dados possui como objetivo buscar informações sobre o perfil dos sujeitos envolvidos (seus conhecimentos sobre informática, a utilização das tecnologias digitais e suas dificuldades, suas concepções sobre RSD e finalidades de utilização da mesma), bem como suas preferências. As questões discursivas são utilizadas para realizar a análise qualitativa do trabalho, baseada na Análise de Conteúdo (BARDIN, 2011 ou WASSERMAN; FAUST, 1994).

Quadro 1 - Questionário

QUESTIONÁRIO AOS IDOSOS

Um Estudo Sobre a Interação de Idosos em Redes Sociais Digitais

Nome:

E-mail:

1. Sexo: ( ) Masculino ( ) Feminino

2. Idade:

( ) 60 a 65 anos

( ) 66 a 70 anos

( ) 71 a 75 anos

( ) 76 a 80 anos

( ) Mais de 80 anos 


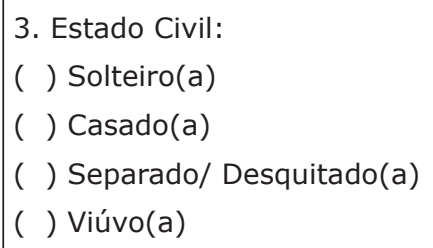

5. Qual sua profissão (exerce ou exercia antes de se aposentar)?

6. Quais são as pessoas com quem reside atualmente?

7. Possui computador em casa? (mesmo que não o utilize). Possui conexão de Internet em casa? (mesmo que não a utilize)
( ) $\operatorname{sim}$
( ) não
( ) $\operatorname{sim}$
( ) não
8. Você utiliza o computador?
( ) $\operatorname{sim}$ ( ) não

9. Caso tenha respondido sim acima, responda:

a) Quantas vezes você utiliza durante a semana?

\section{( ) $1 \mathrm{x} \quad$ ( ) $2 \mathrm{x} \quad$ ( ) $3 \mathrm{x} \quad$ ( ) Mais de 3 vezes}

b) Há quanto tempo você utiliza o computador:

( ) 6 meses ( ) 1ano ( ) 2 anos ( ) mais de 2 anos

c) Por qual motivo você começou a usar o computador? d) Atualmente você utiliza o computador com que idade? Com que finalidade?

( ) Rede social ( ) Pesquisa ( ) Comércio eletrônico

( )Bate-papo ( ) Notícias ( ) E-mail ( ) Jogos Outra(s):

e) Geralmente, você utiliza o computador em que local?

( ) N ( ) Casa ( ) Lan House

10. Caso tenha respondido não acima, responda:

a) Porque não utiliza o computador?

b) Qual a principal dificuldade impede de aprender a utilizar o computador?

11. Quais conhecimentos você possui de informática:

( ) Editor de Texto

( ) Planilha

( ) Internet

( ) Windows

Outro(s):

12. Você sabe o que significa uma Rede Social?

13. Qual Rede Social você utiliza?

( ) Orkut

( ) Facebook

( ) Twitter

( ) Não tenho Rede Social

Outra(s): 
14. Você conhece o Facebook? Qual a sua opinião sobre o Facebook?

15. Caso você utilize o Facebook, responda as perguntas abaixo:

a) Com que frequência utiliza?

( ) Todos os dias

( ) De 4 a 6 vezes na semana

( ) De 1 a 3 vezes na semana

( ) Raramente uso

b) Geralmente acessa o Facebook em que local?

( ) Em casa

( ) Lan House

( ) Dispositivo móvel (celular, smartphone)

Outro(s):

c) Quais os recursos do Facebook que você mais utiliza?

( ) Curtir

( ) Enviar mensagens

( ) Escrever comentários no mural

( ) Conversar Chat

( ) Postar fotos

( ) Jogos

( ) Postar vídeos

( ) Criar eventos

( ) Postar músicas

( ) Aplicativos

Fonte: Elaborado pelo pelas próprias autoras.

O segundo passo consiste no monitoramento dos dados através do qual serão coletadas as interações do grupo de idosos. Essa coleta de dados será efetuada na Application Programming Interface (API) da rede social através do crawler NodeX $L^{3}$ e do aplicativo Netvizz ${ }^{4}$. Segundo Recuero [23], essas ferramentas vêm com crawlers embutidos facilitando a coleta do pesquisador. A escolha por esses sistemas se deve pelo fato de realizarem a coleta mais adequada para o estudo, por buscar os dados públicos diretamente na API do site de rede social e não precisar de um servidor dedicado (RECUERO , 2014). Os dados coletados são utilizados para realizar a análise quantitativa do trabalho, através das métricas para Análise de Redes Sociais baseadas em nós e arestas e na interação. Entre as principais métricas (RECUERO, 2009, PAÚL, 2005, LOPES, 2012, SILVA, 2010):

1) Grau de Centralidade - representa a quantidade de nós que estão interligados a ele. O grau do nó mostra a atividade do nó no grafo, definindo sua importância, bem como sua influência na rede. O grau pode variar de 1 até $\mathrm{N}$. Onde $\mathrm{N}$ é quantidade total de nós no grafo.

2) Diâmetro - o diâmetro é calculado como o maior valor de distância geodésica ${ }^{5}$ entre quaisquer pares de nós pertencentes ao grafo.

3) Densidade - a densidade indica o grau de conexão de uma rede, ou seja, a quantidade de conexões de um grafo $(\mathrm{d}(\mathrm{G})$ ). Para medir a densidade de uma rede, temos que inserir a quantidade de arestas da rede e dividir pela quantida-

\footnotetext{
${ }^{3}$ NodeXL: Network Overview, Discovery and Exploration for Excel - um template open-source para Excel 2007 e 2010. Download disponível em: <http://nodexl.codeplex.com/>

4 Netvizz é uma ferramenta que extrai dados de diferentes seções da plataforma Facebook (perfil pessoal, grupos, páginas) Download disponível em: http://noduslabs.com/radar/ netvizz-export-facebook-network-graph/

${ }^{5}$ Distância geodésica é a menor distância que une dois pontos em um grafo.
} 
de máxima de arestas suportadas por essa rede. A densidade da rede pode variar entre 0 e 1 . A rede, com máxima conexão possui densidade igual a 1 .

4) Centralidade de Closeness (Proximidade) - descreve a sua independência em relação aos outros nós da rede, ele é tão mais central quanto menor o caminho que ele precisa percorrer para alcançar os outros nós da rede. Reflete que quanto mais próximo o nó encontra-se em relação aos demais nós da rede, mais rápida a interação. Essa métrica é calculada pelo inverso da soma das distâncias geodésicas de um nó em relação aos demais nós do grafo.

5) Centralidade de Betweenness (Intermediação) - descreve a localização global do nó na rede, levando em consideração os relacionamentos indiretos. Reflete o quanto o nó é intermediador facilitando o fluxo de informação na rede. É calculado pela soma das distâncias geodésicas entre quaisquer dois nós que passam pelo nó analisado.

6) Centralidade de Eigenvector (Autovetor) - descreve a relevância desse nó em função de sua relação com os nós da rede. Se um nó está ligado a outros que se encontram em uma posição central da rede, o nó terá centralidade de autovetor alta. Um nó com alto valor de centralidade de autovetor possui grande probabilidade de transmitir o fluxo de informação para os outros elementos de forma indireta.

7) Coeficiente gigante - o coeficiente gigante $(g c)$ é calculado com base no componente gigante $(m c)$ de uma rede. Representa o componente conectado com o maior número de nós.
8) Coeficiente de Clusterização - o coeficiente de clusterização de um nó cc $\left(n_{i}\right.$ ) é a razão entre o número de arestas entre vizinhos do nó $n_{i}$ e o número máximo de arestas possíveis entre os vizinhos do nó $n_{i}$. Expressa a probabilidade de dois vértices que estão conectados possuírem uma conexão em comum com um terceiro vértice.

Por fim, a fase de análise de dados é feita de forma qualitativa e quantitativa. Referente a análise quantitativa, é importante ressaltar que a proposta apresenta como diferencial a coleta dos dados diretamente na API do site de rede social através de ferramentas computacionais. Além disso, permite gerar grafos contendo as interações dos sujeitos da pesquisa que serão analisados através das métricas para análise de redes sociais, possibilitando descobrir as formas como ocorre a interação entre essas pessoas no meio digital.

\section{Resultados}

O Facebook foi a rede social selecionada a ser estudada. O principal motivo deve-se ao fato do seu rápido crescimento no número de usuários tanto no Brasil (COMSCORE, 2012a) como globalmente (COMSCORE, 2012b). Além disso, segundo o Relatório Brazil Digital Future in Focus ${ }^{6}$, publicado em março de 2013, o qual analisa as principais tendências no uso da internet, as mídias sociais capturaram a maior porcentagem do tempo dos usuários de internet no Brasil com um crescimento de $167 \%$ quando comparada com outros conteúdos, como notícias/informações, jogos, entretenimento e serviços. Os brasileiros estão altamente enga-

Disponível em:<http://www.comscore.com/por/Insights/ Press_Releases/2013/3/comScore_Releases_2013_Brazil_DigitalFuture_in_Focus_Report> 
jados em conteúdo social com média de tempo conectado em sites de redes sociais correspondente a quase 10 horas de permanência. Com relação à faixa etária, nota-se que o número de pessoas com mais de 50 anos na Web aumentou 223\% entre 2005 e 2011, chegando a 8,1 milhões de usuários. O Facebook apresenta o maior número de usuários contendo a preferência da audiência tanto no Brasil quanto no mundo. Representa também a plataforma mais utilizada pelos brasileiros idosos, dessa forma, foi a rede social digital escolhida como objeto deste estudo.

Referente ao passo 1 da metodologia, o questionário foi aplicado a um grupo composto de 27 idosos pertencentes a uma organização social? ${ }^{7}$. Nesta empresa, os sujeitos da pesquisa, todos do sexo feminino, realizam várias atividades como: artesanato, dança, ginástica e aulas de espanhol. Como existem poucos grupos de idosos na cidade de XXXX este foi o grupo que aceitou participar do estudo.

As respostas dos participantes as perguntas fechadas do questionário foram utilizadas para geração de gráficos. A partir dos gráficos gerados foi possível constatar com relação ao perfil dos sujeitos da pesquisa que a idade predominante dos idosos foi entre os 66 e 70 anos (33\%), a maior parte dos entrevistados eram viúvas $(44 \%)$ e a maioria possuía ensino médio completo (33\%).

Com relação a informática, a maior parte dos participantes possuía computador em casa $(85 \%)$, conexão de internet $(78 \%)$, utilizava o computador (70\%) mais de 3 (três) vezes durante a semana ( $89 \%)$ e por um tempo superior a 2 (dois) anos (67\%). A principal fina-

\footnotetext{
${ }^{7}$ A organização social de direito privado, sem fins lucrativos, tem por atribuição proporcionar o bem-estar e qualidade de vida aos seus trabalhadores e as suas famílias. Porém, não permite a divulgação do seu nome devido à política de privacidade dessa empresa. Dessa forma, mantêm-se o anonimato do grupo de idosos analisado.
}

lidade para utilização do computador foi a rede social $(21 \%)$ seguido da pesquisa (20\%) e o principal conhecimento em informática o uso da internet (37\%).

Quanto a RSD, o Facebook obteve a preferência ( $53 \%$ ) dos entrevistados, sendo que a maioria o utiliza todos os dias ( $56 \%)$, em suas residências (89\%) ou através de dispositivo móvel $(11 \%)$ e o principal recurso utilizado é o envio de mensagens (24\%).

\subsection{Análise Qualitativa}

A análise qualitativa foi realizada seguindo as três fases cronológicas descritas na Análise de Conteúdo (BARDIN, 2011 ou WASSERMAN; FAUST, 1994). Na primeira fase, a pré-análise, caracterizada pela organização dos dados, foi realizada uma leitura flutuante dos questionários procurando se conhecer todo o texto. Logo após, foram selecionadas como documento para análise as respostas descritas pelo público-alvo da pesquisa as perguntas abertas do questionário. Para análise do conteúdo das mensagens foi selecionado o sistema de categorias e como critério de categorização semântico o tema, com o objetivo de descobrir a motivação para os idosos interagirem nas RSD, bem como as razões que os impedem de utilizar o computador. Segundo Bardin (2011), o tema é utilizado como unidade de registro para estudar motivações de opiniões, de atitudes, de valores, de crenças, etc.

Para as respostas relacionadas aos idosos que utilizam o computador foram definidas como hipótese a priori as seguintes categorias: socialização e comunicação. Já para as respostas relacionadas aos idosos que não utilizam o computador foram definidas a priori as seguintes categorias: medo e desconhecer. Cada pergunta, com suas respectivas respos- 
tas foram anotadas em uma tabela, visando facilitar a visualização dos contrastes.

A segunda fase, exploração do material, consistiu na aplicação do que foi estabelecido na pré-análise. Foram realizadas operações de codificação por classificação, transformando os dados brutos das mensagens em unidades de registro semânticas (categorias temáticas). Os elementos foram classificados em categorias, tendo em vista o que cada um deles tem em comum com outros. O que permitiu seu agrupamento foi a parte comum existente entre eles.

Na última fase, tratamento dos resultados, com relação as respostas as perguntas abertas aos idosos que utilizam o computador, bem como aos que não utilizam o mesmo, foram construídas tabelas contendo o sistema de categorização. Outras categorias, além das definidas a priori, foram criadas. A pergunta 1 além da categoria comunicação, tem-se e atualização e atividades extras. A categoria atualização mostrou-se como a principal motivação para os idosos começarem a utilizar o computador, seguida pela comunicação e atividades extras. Na pergunta 2, foi necessário criar a categoria utilização. A significação das redes sociais pelo público da pesquisa representa principalmente a comunicação seguida pela socialização e utilização. Na pergunta 3 , foram criadas as categorias aspectos positivos e aspectos negativos. A principal opinião com relação ao site de rede social $\mathrm{Fa}$ cebook foi ressaltada através dos aspectos positivos seguida pela socialização, comunicação, utilização e aspectos negativos.

Com relação as respostas as perguntas abertas aos idosos que não utilizam o computador, também foram construídas tabelas contendo as categorias. Outras categorias, além das definidas a priori, foram criadas. Na pergunta 4 foi criada a categoria não possuir e a emocional. O principal motivo para os idosos não utilizarem o computador é o desconhecer, seguido pelo fato de não possuir um computador e a motivação emocional. A pergunta 5 também constatou que a principal dificuldade para não utilizar o computador é o desconhecer seguido pelo medo e o fato de não possuir.

É importante salientar que com relação a pergunta aberta de número 7 - Quais são as pessoas com que reside atualmente? - a maioria das idosas da pesquisa respondeu que mora sozinha.

Através da aplicação das perguntas fechadas de múltipla escolha do questionário foi possível identificar o público-alvo da pesquisa, bem como suas preferências. Os aspectos principais obtidos através desse método de coleta dos dados foram que a maioria dos idosos que participaram da pesquisa possuía entre 66 e 70 anos de idade e a principal finalidade para utilização do computador era o acesso a rede social Facebook. Além disso, a maioria dos idosos residia sozinho e acessava o Facebook todos os dias para o envio de mensagem.

A análise das respostas as perguntas abertas aos idosos que utilizam o computador permitiram descobrir que a principal motivação para os idosos começarem a utilizarem o computador foi a necessidade de manterem-se atualizados a nova realidade através do uso da tecnologia. Ressalta, dessa forma, o conteúdo do artigo $21^{\circ}$ do Estatuto do Idoso [9], o qual assegura o direito aos idosos ao aprendizado das técnicas de computação e avanços tecnológicos visando a sua integração a vida moderna. Outra motivação destacada pelos idosos foi a comunicação, sendo que ambas motivações (atualização e comunicação) estão presente na interação do ser humano com o mundo digital. As pessoas interagem comunicando-se e 
expressando suas ideias e opiniões, e ao atualizarem-se estão recebendo conhecimento e aprendendo umas com as outras.

Além disso, as redes sociais representam aos idosos principalmente a comunicação seguida pela socialização reforçando os conceitos contidos na teoria sócio-interacionista. Para Vygotsky (apud CASTELLS, 2003), a socialização proporciona interação com o outro ser social através do uso da linguagem (comunicação) sendo de extrema importância para o aprendizado e desenvolvimento humano.

Por fim, a categoria aspectos positivos destacados na opinião sobre o site de rede social Facebook reafirma que a sua utilização pelos idosos traz inúmeros benefícios a essa população. Entre as respostas descritas, pode-se destacar: ótimo, indispensável, utilíssimo, para conversar, fazer amigos, postar fotos. Outra categoria destacada foi a socialização, aspecto também muito importante ao seu interagir de forma mútua no meio digital.

Com relação a análise das respostas as perguntas abertas aos idosos que não utilizam o computador foi possível compreender suas dificuldades com relação a tecnologia digital. A principal dificuldade para não utilizar o computador foi o desconhecimento, confirmando a maior necessidade de políticas governamentais que assegurem o direito aos idosos ao acesso as tecnologias da computação. Portanto, a relação entre os aspectos biológicos, psicológicos e sociais do envelhecimento que contribuem para que cada idoso interaja com a sociedade de uma forma única e heterogênea não constituem fatores que impeçam a utilização do computador.

\subsection{Análise Quantitativa}

A análise quantitativa é realizada após o monitoramento dos dados através da aplicação das métricas das redes sociais baseadas em nós e arestas e na interação. É necessário salientar que o público-alvo da pesquisa é composto de vinte e sete idosos, sendo que dezenove pessoas utilizavam o computador e oito pessoas não. Dessas dezenove pessoas que utilizavam o computador, dezesseis idosos utilizavam o site de rede social Facebook. No monitoramento dos dados foram convidados para participar todos que utilizavam o Facebook. Porém devido as questões adversas dos dezesseis convidados, somente dez compareceram. Portanto, foram aplicadas as métricas para as Redes Sociais para dez pessoas e apresentados os resultados quanto a análise quantitativa.

Para a realização do cálculo das métricas baseada em nós e arestas, primeiramente foi efetuada a coleta dos dados no Facebook pelo crawler Netvizz e utilizado como software para análise o $\mathrm{Gephi}^{8}$. Como para cada métrica de rede social os sujeitos foram analisados de forma isolada, sendo muito extensa a apresentação de todos os resultados com imagem foi selecionado o sujeito 6 e a métrica de Clustering. Sendo assim, o Quadro 2 apresenta a coleta com a ferramenta Netvizz (rede pessoal), a métrica de Clustering aplicando o algoritmo ForceAtlas evitando a sobreposição dos nós e a análise dos dados obtidos para o sujeito 6 .

${ }_{8}^{8}$ Disponível em: <http://gephi.github.io/> 
Quadro 2 - Coeficiente de Clustering Baseada em Nós - Sujeito 6

\begin{tabular}{|c|c|c|c|}
\hline $\begin{array}{c}\text { Sujei- } \\
\text { to }\end{array}$ & $\begin{array}{c}\text { Coleta dos } \\
\text { Dados }\end{array}$ & $\begin{array}{c}\text { Aplicação } \\
\text { Métrica }\end{array}$ & \multicolumn{1}{c|}{$\begin{array}{c}\text { Análise dos } \\
\text { Dados }\end{array}$} \\
\hline 6 & & & $\begin{array}{l}\text { Neste grafo forma- } \\
\text { ram-se sete clus- } \\
\text { ters. Destacam-se } \\
\text { o cluster em mar- } \\
\text { rom que representa } \\
\text { a família da idosa } \\
\text { e o em azul escu- } \\
\text { ro, que são as ido- } \\
\text { sas da pesquisa. O } \\
\text { cluster azul escuro } \\
\text { apresenta a pessoa } \\
\text { com maior número } \\
\text { de conexões. }\end{array}$ \\
\hline
\end{tabular}

Fonte: Elaborado pelo pelas próprias autoras.

A Tabela 1 apresenta um resumo com os principais valores obtidos utilizando os cálculos das métricas de redes sociais para cada um dos sujeitos e também os dados da rede completa com todos os sujeitos da pesquisa. Os valores que se destacaram estão em negrito e são descritos deforma detalhada.
No grau de centralidade destacou-se a rede do sujeito de número 2 que apresentou o maior grau, com 116 conexões, representado por uma pessoa da família do sujeito. A rede do sujeito de número 3 que apresentou o nó com maior grau, com 48 conexões, representado por uma idosa da pesquisa, e também a rede do sujeito número 6 , que o nó com maior grau, com 27 conexões, foi de uma idosa da pesquisa. Na métrica de diâmetro destacou-se a idosa de número 6, com diâmetro maior igual a 9 e a idosa de número $4 \mathrm{com}$ densidade maior de 0,416 . Na centralidade de closeness destacou-se a rede do sujeito de número 2, por apresentar, aliada a menor distância, quantidade elevada de nós com distância mínima, apresentando maior capacidade de interação. $\mathrm{Na}$ centralidade de betweenness destacaram-se a rede do sujeito de número 3 , de número 5 , por além de apresentarem valores mais altos de intermediação $(5.116,8$ e $3.806,22)$ entre os nós com valores mais altos serem representados por idosas da pesquisa.

Tabela 1 - Resumo das Métricas de Redes Sociais

Baseada em Nós

\begin{tabular}{|l|l|l|l|l|l|l|l|l|}
\hline $\begin{array}{l}\text { Su } \\
\text { jei } \\
\text { tos }\end{array}$ & $\begin{array}{l}\text { Grau de } \\
\text { Centra } \\
\text { lidade }\end{array}$ & $\begin{array}{l}\text { Diâme } \\
\text { tro }\end{array}$ & $\begin{array}{l}\text { Densi } \\
\text { dade }\end{array}$ & $\begin{array}{l}\text { Centralidade } \\
\text { Closeness }\end{array}$ & $\begin{array}{l}\text { Centralidade } \\
\text { Betweenness }\end{array}$ & $\begin{array}{l}\text { Centra } \\
\text { lidade } \\
\text { Eigenvector }\end{array}$ & $\begin{array}{l}\text { Coefi cien- } \\
\text { te } \\
\text { Gigante }\end{array}$ & $\begin{array}{l}\text { Coefi } \\
\text { ciente } \\
\text { Clustering }\end{array}$ \\
\hline 1 & 46 & 5 & 0,128 & 1 até 3,848 & 0 até $1.147,3$ & 0 até 1 & - & 5 \\
\hline 2 & $\mathbf{1 1 6}$ & 5 & 0,072 & $\mathbf{0}$ até $\mathbf{3 , 6 8 5}$ & 0 até $8.257,9$ & 0 até 1 & - & 6 \\
\hline 3 & $\mathbf{4 8}$ & 5 & 0,073 & 0 até 3,8140 & $\mathbf{0}$ até $\mathbf{5 . 1 1 6 , 8}$ & 0 até 1 & - & 7 \\
\hline 4 & 22 & 3 & $\mathbf{0 , 4 1 6}$ & 0 até 2,6 & 0 até 125,04 & 0 até 1 & - & - \\
\hline 5 & 59 & 8 & 0,076 & 0 até 5,38 & $\mathbf{0}$ até $\mathbf{3 . 8 0 6 , 2 2}$ & 0 até 1 & - & 7 \\
\hline 6 & $\mathbf{2 7}$ & $\mathbf{9}$ & 0,036 & 0 até 6,48 & 0 até $3.408,46$ & 0 até 1 & - & $\mathbf{7}$ \\
\hline 7 & 78 & 6 & 0,053 & 0 até 4,89 & 0 até $9.026,76$ & 0 até 1 & - & $\mathbf{7}$ \\
\hline 8 & 66 & 5 & 0,148 & 1,52 até 3,70 & 0 até 355,38 & $\mathbf{0}$ até $\mathbf{1}$ & - & 3 \\
\hline 9 & 89 & 3 & 0,239 & 1,10 até 2,87 & 0 até $1.411,11$ & 0 até 1 & - & - \\
\hline 10 & 52 & 8 & 0,106 & 0 até $\mathbf{4 , 8 3}$ & 0 até $1.136,24$ & 0 até 1 & - & $\mathbf{1}$ \\
\hline $1-10$ & 116 & 10 & 0,011 & 0 até 7,54 & $\mathbf{0}$ até $\mathbf{6 9 . 0 6 8 , 9 7}$ & 0 até 1 & $\mathbf{1 0 6 5}$ & $\mathbf{1 0}$ \\
\hline
\end{tabular}

Fonte: Elaborada pelo pelas próprias autoras.
Além disso, a rede completa (1-10) apresentou entre os nós com mais alto valor de intermediação 4 idosas pertencentes ao grupo analisado. Na centralidade de eigenvector, 
destacou-se a rede do sujeito de número 8 por apresentar maior quantidade de nós com valores de autovetores altos.

Também é importante ressaltar o coeficiente gigante da rede completa (1-10), com todas as idosas da pesquisa, que apresentou também um valor elevado. No coeficiente de clustering destacou-se a rede do sujeito de número 6, contando com sete clusters, na qual a pessoa com maior número de conexões pertencia ao cluster das idosas da pesquisa. A rede do sujeito de número 7 , contando com sete clusters, sendo o cluster mais numeroso representado pelas idosas da pesquisa. A rede completa (110) além de apresentar um número elevado de clusters, o cluster mais numeroso foi representado pelas idosas da pesquisa.

Para a realização do cálculo das métricas baseadas na interação, primeiramente foi efetuada a coleta dos dados na API do Facebook pelo crawler NodeXL, o qual também foi utilizado como software para análise. É necessário salientar, que o software não possui opção para anexar grafos como no Gephi, ou outra opção semelhante que desempenhe essa função. Sendo assim, não foi possível gerar a rede completa com todos os sujeitos da pesquisa.

Como para cada métrica de rede social os sujeitos foram analisados de forma isolada, sendo muito extensa a apresentação de todos os resultados obtidos com imagem foi selecionado o sujeito 7 e a métrica de Clustering para apresentação das imagens obtidas. Sendo assim, o Quadro 3 apresenta a coleta com a ferramenta NodeXL, para geração da imagem foi selecionado a importação das postagens no Facebook referentes ao período de 02/10/2014 à 09/10/2014. Segundo Nascimento [30] o usuário pode criar grupos baseado em algum atributo dos nós ou a partir de componentes fortemente conectadas encontradas pelo NodeXL. Outra forma de agrupamento ocorre através de algoritmos de agrupamento: WakitaandTsurumi, Girvan-Newman e Clauset-Newman-Moore. Estes algoritmos quando aplicados tentam posicionar os nós analisando o conjunto de nós mais fortemente conectados e separando-os em grupos. No Quadro 3 foi aplicado o coeficiente de Clustering e utilizado o algoritmo de agrupamento Girvan-Newman.

Quadro 3 - Coeficiente de Clustering Baseada na Interação- Sujeito 7

\begin{tabular}{|c|c|c|c|}
\hline $\begin{array}{l}\text { Sujei- } \\
\text { to }\end{array}$ & $\begin{array}{c}\text { Coleta dos } \\
\text { Dados }\end{array}$ & $\begin{array}{c}\text { Aplicação mé- } \\
\text { trica }\end{array}$ & $\begin{array}{c}\text { Análise } \\
\text { dos Dados }\end{array}$ \\
\hline 7 & & 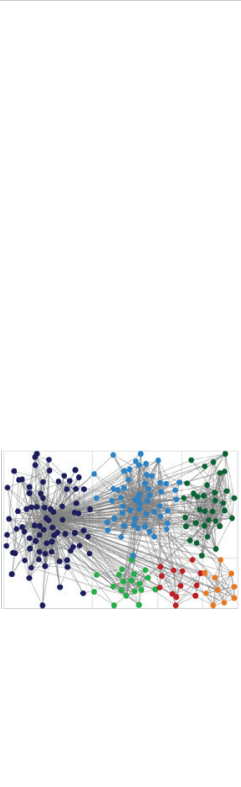 & $\begin{array}{l}\text { Neste } \\
\text { grafico for- } \\
\text { maram-se } \\
6 \text { clusters. } \\
\text { Destaca- } \\
\text { ram-se } \\
\text { o clus- } \\
\text { ter mais } \\
\text { numeroso } \\
\text { em azul } \\
\text { escuro com } \\
80 \text { nós, } \\
\text { que são as } \\
\text { idosas da } \\
\text { pesquisa e } \\
\text { o azul claro } \\
\text { que é a } \\
\text { família da } \\
\text { idosa. }\end{array}$ \\
\hline
\end{tabular}

Fonte: Elaborado pelo pelas próprias autoras.

A Tabela 2 apresenta um resumo com os principais valores obtidos utilizando os cálculos das métricas de redes sociais para cada um dos sujeitos. Os valores que se destacaram estão em negrito e são descritos de forma mais detalhada.

No grau de centralidade destacou-se a rede do sujeito de número 7 que apresentou o 
maior grau, com 212 conexões, representado pelo sujeito da pesquisa, e também o número mais elevado de interações de amizade, com 1384 relações. A rede do sujeito de número 1 , que apresentou a maior variedade de interações com 5 tipos diferentes. Na métrica de diâmetro destacou-se a idosa de número 2 , com diâmetro maior igual a 6, sendo que a maioria das idosas (oito) apresentaram os menores diâmetros, com valor igual a 2. Na métrica da densidade a idosa de número 9 apresentou a rede com maior densidade, igual a 0,254.

$\mathrm{Na}$ centralidade de closeness como os resultados dos valores foram muito próximos destacaram-se a rede do sujeito de número 2, por apresentar uma variação de 0 até 0,005 e o sujeito da pesquisa bem como as idosas da pesquisa apresentarem o valor de centralidade igual a 0,002, e também a rede do sujeito de número 7 que apresentou uma variação de 0,002 até 0,005 e as idosas da pesquisa apresentarem o valor de centralidade entre 0,002 e 0,003 , pois quanto menor o valor, maior a capacidade de interação. Na centralidade de betweenness destacaram-se a rede dos sujeitos de número 6 e 7, por apresentarem valores mais altos de intermediação $(18076,835 \mathrm{e}$ $17647,219)$, participando de forma mais ativa no processo de interação. Na centralidade de eigenvector, destacou-se a rede do sujeito de número 1 , por apresentar maior quantidade de nós com valores de autovetores altos e o sujeito da pesquisa também apresentar um valor alto.

No coeficiente de clustering destacou-se a rede do sujeito de número 5 , contando com o maior número de clusters, com oito clusters, sendo o cluster das idosas da pesquisa bem numeroso. A rede do sujeito de número 7, contando com seis clusters, sendo o cluster mais numeroso representado pelas idosas da pesquisa.
Tabela 2 - Resumo das Métricas de Redes Sociais Baseadas na Interação

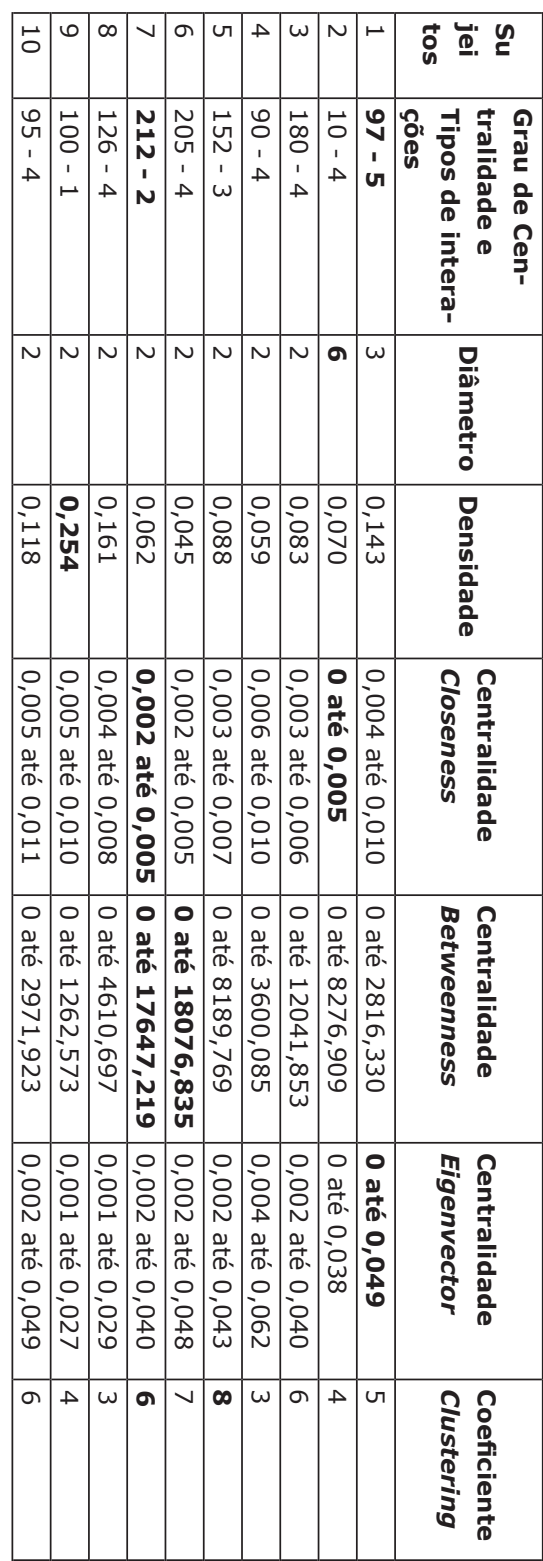

Fonte: Elaborada pelo pelas próprias autoras.

É importante relatar que durante a realização desse trabalho foram constatadas algumas dificuldades, tanto na parte da coleta como nos softwares utilizados. As ferramentas computa- 
cionais utilizadas na pesquisa não permitiam o envio dos dados de forma virtual. Como o local da organização social era bloqueado o acesso ao Facebook, foi necessário a utilização de uma conexão de Internet própria para coleta dos dados do grupo de idosos. Além disso, foi necessário marcar um dia com todas as idosas que utilizavam a RSD, para que as mesmas, de forma individual, se conectassem ao Facebook, através do seu nome de usuário e senha, autorizando, dessa forma, a coleta dos dados tanto para o software Netvizz como para o Nodexl.

\section{Considerações Finais}

A utilização das redes sociais é um fenômeno que está em constante crescimento pela sociedade desde a última década. Por sua vez, as redes sociais na Web apresentam muitos aspectos de comunicação e interação entre o homem e o computador, agregado a sua ampla aceitação e crescente aumento no número de usuários.

O envelhecimento da população brasileira é um fator que evidencia uma maior necessidade de preocupação com a relação que estas pessoas estabelecem com o mundo. Na vida social das pessoas idosas, observa-se a tendência crescente ao isolamento devido às mudanças sociais como o desligamento do ambiente de trabalho. Essa situação é, muitas vezes, aliada a problemas de saúde ou de deslocamento, contribuindo para agravar esse processo de solidão.

Nesse caso, a análise das interações sociais na rede social é em função da comunicação, proporcionando que a população crescente de idosos no Brasil seja analisada no contexto digital. Como consequência, os idosos podem estabelecer ou aprimorar as relações sociais de forma simples e rápida, e em muitos casos, algumas pessoas idosas possam sair do isolamento, com um envelhecimento bem-sucedido.

Além disso, os idosos, ao longo do seu processo de envelhecimento, passam por inúmeras mudanças biológicas, sociais e psicológicas. Dentre estas, a senescência e a mudança dos papéis sociais, fases naturais na vida do idoso, mas que dependendo do processo de adaptação individual podem contribuir de forma negativa, causando o isolamento dessa faixa etária pela diminuição de suas funções biológicas e sociais.

Por outro lado, a interação social contribui para a aprendizagem e o consequente desenvolvimento humano em qualquer fase da vida do ser humano, principalmente através do uso da linguagem, que serve como instrumento mediador para a comunicação entre as pessoas (CASTELLS, 2003). Associado a esse fato, a comunicação e a aquisição de conhecimento no meio digital, através do Facebook constitui uma das formas de manter a função cognitiva dos idosos, contribuindo para um envelhecimento bem-sucedido. No meio digital, a RSD Facebook desponta como uma ferramenta social que permite ampliar as formas de comunicação e compartilhamento de informações da sociedade através do uso da tecnologia. Demostra, dessa forma, a necessidade de preocupação de avaliar-se como é realmente a interação que as pessoas idosas estabelecem com o mundo tecnológico através do uso da RSD.

Portanto, a proposta deste trabalho foi realizar um estudo, tanto quantitativo como qualitativo, das interações sociais dos idosos em uma RSD. Sendo assim, buscou, através dos resultados obtidos com essa pesquisa, verificar as preferências e dificuldades desse gru- 
po no meio digital, com a intencionalidade de aproveitar a dinâmica da plataforma digital para promoção da interação social. Interação vivenciada com outras pessoas e também com o mundo tecnológico, a qual se faz extremamente necessária para o auxílio desse grupo tão especial, que encontra-se em crescimento e possui uma larga experiência de vida para ser compartilhada, aliada aos cuidados advindos de uma idade avançada.

Através dos dados obtidos e analisados neste trabalho, concluiu-se que foi alcançado os objetivos iniciais propostos. A análise qualitativa foi realizada através do estabelecimento de categorias de análise e a quantitativa foi obtida as ações mais realizadas na RSD através de ferramentas computacionais. Dessa forma, foi possível melhor compreender os gostos, preferências e dificuldades dos idosos na sua relação com o meio digital. Sendo assim, a dinâmica do Facebook pode ser empregada como uma ferramenta on-line para aprimorar as relações sociais dos idosos contribuindo para um envelhecimento bem-sucedido.

Através da correlação entre a análise qualitativa e quantitativa foi possível constatar que as abordagens se complementaram. As categorias comunicação e socialização foram as que mais se destacaram na abordagem qualitativa. A comunicação, além de constituir uma das motivações para os idosos utilizarem o computador, também representa a significação das redes sociais para o público da pesquisa seguida pela socialização e a opinião sobre o site de rede social Facebook. Por sua vez, na abordagem quantitativa essas categorias foram reafirmadas porque se constatou que os idosos realmente interagem no meio virtual, socializando-se, construindo conhecimento através das diferentes interações realizadas, e se comunicando principalmente com familiares e amigos.

Além disso, constatou-se que as pessoas idosas que participam de um grupo, através do qual se conhecem fisicamente e inclusive realizam atividades recreativas juntas, possuem interação social no meio virtual. Desta forma, surge o seguinte questionamento: Grupos de idosos nos quais não existe interação física, interação virtual poderá existir?

Outra possibilidade de trabalho futuro seria aproveitar os dados obtidos com relação as preferências dos idosos na interação da rede social, e associar um estudo voltado para as dificuldades advindas com o processo da senescência e construir uma RSD voltada especificamente para o público idoso ou uma adaptação do próprio Facebook para o público idoso. A principalmente dificuldade para os idosos não utilizarem as RSDs foi a necessidade do aprendizado de como utilizar. Assim, se a plataforma digital fosse voltada para a necessidade dos idosos, talvez não houvesse esse impedimento em sua utilização, e mais pessoas idosas estariam conectadas as redes sociais digitais. 


\section{Referências}

BARDIN, L. Análise de Conteúdo. Lisboa: Ed. 70, 2011.

BENEVENUTO, F.; ALMEIDA, J.; SILVA, A. Explorando Redes Sociais On-line: da coleta, análise de grandes bases de dados às aplicações. In: SIMPÓSIO BRASILEIRO DE REDES DE COMPUTADORES E SISTEMAS DISTRIBUÍDOS, 29., 2011, Campo Grande, BR-MS. [Anais]. Campo Grande, MS: SBRC, 2011. Cap. 2.

BLATTMANN, U.; SILVA, F.C.C.D. Colaboração e Interação na Web 2.0 e Biblioteca 2.0. Revista ACB: Biblioteconomia em Santa Catarina, Florianópolis, v. 12, n. 2, p. 191-215, dez. 2007.

BRANDÃO, M.D.F.M.; SILVEIRA, R.M.H. O Orkut e a Velhice: comunidades e discursos. In: COUTO, E.S.; ROCHA, T.B. (Org.). A Vida no Orkut: narrativas e aprendizagens nas redes sociais. Salvador: EDUFBA, 2010. P. 165-188.

BRASIL. Lei no $\mathbf{1 0 . 7 4 1}$, de $1^{\circ}$ de outubro de 2003. Dispõe sobre o Estatuto do Idoso e dá outras providências Art. 48, conforme a Lei no 8.842. Brasília, 2003.

CASTELLS, M. A Galáxia da Internet: reflexões sobre a Internet, os negócios e a sociedade. Rio de Janeiro: Zahar, 2003.

COMSCORE. Facebook Blasts into Top Position in Brazilian Social Networking Market Following Year of Tremendous Growth. [S.I.], 2012a. Disponível em: <http://www.comscore.com/Insights/Press_Releases/2012/1/Facebook_Blasts_into_Top_Position_in_Brazilian_Social_Networking_Market> Acesso em: 21 abr. 2013.

COMSCORE. Facebook Shows Strong Growth Over Past Five Years. [S.I.], 2012b. Disponível em: <http://www.comscoredatamine.com/2012/02/facebook-shows-strong-growth-over-past-five-years> Acesso em: 19 abr. 2013.

DOLL, J. Bem-Estar na Velhice: mitos, verdades e discursos ou a gerontologia na modernidade. Revista Brasileira de Ciências do Envelhecimento Humano, Passo Fundo, v. 3, n. 1, p. 9-21, jan./jun. 2006.

FRAgoso, S.; ReCuero, R.; AMARAL, A. Métodos de Pesquisa Para Internet. Porto Alegre: Sulina, 2011.

IBGE. Sala de Imprensa. [site]. Rio de Janeiro, 2011. Disponível em: <http://saladeimprensa.ibge. gov.br/iwnoticias?view=noticia\&id=1\&busca=1\&idnoticia=1866> Acesso em: 21 jun. 2013. 
IBGE. Projeção da População do Brasil por Sexo e Idade: 2000-2060. Rio de Janeiro, 2013. Disponível em: <http://www.ibge.gov.br/home/estatistica/populacao/projecao_da_populacao/2013/> Acesso em: 10 ago. 2013.

JANTSCH, A. et al. As Redes Sociais e a Qualidade de Vida: os idosos na era digital. IEEE-RITA, Madrid, v. 7, n. 4, p. 173-179, nov. 2012. Disponível em: <http://rita.det.uvigo.es/201211/uploads/ IEEE-RITA.2012.V7.N4.A2.pdf> Acesso em: 19 ago. 2013.

LEWIS, S.; ARIYACHANDRA, T. Seniors and On-line Social Network Use. Journal of Information Systems Applied Research, Chicago, v. 4, n. 2, p. 4-18, 2011. Disponível em: <http://jisar.org/2011-4/ N2/JISARv4n2p4.pdf> Acesso em: 7 jan. 2014.

LOPES, G.R. Avaliação e Recomendação de Colaborações em Redes Sociais Acadêmicas. 2012. Tese (Doutorado) - Programa de Pós-Graduação em Computação, Instituto de Informática, Universidade Federal do Rio Grande do Sul, 2012, Porto Alegre, BR-RS.

MORAES, E.N.; MORAES, F.L.; LIMA, S.D.P.P. Características Biológicas e Psicológicas do Envelhecimento. Revista Medicina de Minas Gerais, Belo Horizonte, v. 20, n, 1, p. 67-73, fev. 2010.

NASCIMENTO, C.S. Pandora: uma ferramenta para visualização incremental e análise de redes sociais acadêmicas. 2013. (Mestrado em Tecnologia) - Programa de Pós Graduação em Tecnologia, Centro Federal de Educação Tecnológica Celso Suckow da Fonseca, 2010, Rio de Janeiro, BR-RJ.

MORAES, E.N.; MORAES, F.L.; LIMA, S.D.P.P. Características Biológicas e Psicológicas do Envelhecimento. Revista Medicina de Minas Gerais, Belo Horizonte, v. 20, n, 1, p. 67-73, fev. 2010.

NERI, A.L. Saúde e Envelhecimento: prevenção e promoção. 1. ed. Brasília: [s.n.], 2009. P. $103-110$.

PÁSCOA, G.M.G. O Contributo da Web Social - rede social Facebook - para a promoção do envelhecimento ativo: estudo de caso realizado na USALBI. 2012. Dissertação (Mestrado) - Instituto Superior de Ciências Sociais e Políticas, Universidade Técnica de Lisboa, 2012, Lisboa, PT. Disponivel em: <http:// hdl.handle.net/10400.5/4427> Acesso em: 16 jul. 2013.

PAÚL, C. Envelhecimento Activo e Redes de Suporte Social. In: PAÚL, C. Sociologia. [S.I.: s.n.], 2005. P. 275-287.

PRIMO, A. Interação Mútua e Interação Reativa: uma proposta de estudo. Revista Famecos, Porto Alegre, n. 12, p. 81-92, 2000. 
PRIMO, A. Interação mediada por computador: comunicação, cibercultura, cognição. Porto Alegre: Sulina, 2007.

RECUERO, R. Contribuições da Análise de Redes Sociais Para o Estudo das Redes Sociais na Internet: o caso da hashtag\#TamojuntoDilma e \#CalaabocaDilma. Revista Fronteiras: estudos midiáticos, São Leopoldo, v. 16, n. 2, p. 60-77, 2014.

RECUERO, R. Redes Sociais na Internet. Porto Alegre: Sulina, 2009.

SCHNEIDER, R.H.; IRIGARAY, T.Q. O Envelhecimento na Atualidade: aspectos cronológicos, biológicos, psicológicos e sociais. Estudos de Psicologia, Campinas, v. 25, n. 4, p. 585-593, out./nov. 2008.

SILVA, T.S.A. Um Estudo de Medidas de Centralidade e Confiabilidade em Redes. 2010. Dissertação (Mestrado em Tecnologia) - Programa de Pós Graduação em Tecnologia, Centro Federal de Educação Tecnológica Celso Suckow da Fonseca, 2010, Rio de Janeiro, BR-RJ.

VYGOTSKY, L.S. Formação Social da Mente. 6. ed. São Paulo: Martins Fontes, 1998.

WASSERMAN, S.; FAUST, K. Social Network Analysis: methods and aplications. Cambridge, UK: Cambrridge University Press, 1994.

WASSERMAN, C. et al. Redes Sociais: um novo mundo para os idosos. RENOTE: novas tecnologias na educação, Porto Alegre, v. 10, jul. 2012. Disponível em: <http://seer.ufrgs.br/index.php/renote/article/ view/30863/19222> Acesso em: 18 mar. 2015.

WEITZEL, L.C.D.S. Abordagem Baseada na Análise de Redes Sociais Para a Estimativa da Reputação de Fontes de Informação em Saúde. 2013. Tese (Doutorado) - Programa de Pós-Graduação em Computação, Instituto de Informática, Universidade Federal do Rio Grande do Sul, 2013, Porto Alegre, BR-RS.

Submetido para avaliação em 26 de março de 2015. Aprovado para publicação em 12 de agosto de 2015.

Lucélia Moreira Chepe - Universidade Federal do Rio Grande, Rio Grande, BR-RS. E-mail: chepe.lucelia@gmail.com Diana Francisca Adamatti - Universidade Federal do Rio Grande, Rio Grande, BR-RS. E-mail: dianaada@gmail.com 